\title{
Direct Backward/Forward Sweep Algorithm for Solving Load Power Flows in AC Droop-Regulated Microgrids
}

\author{
Guzmán Díaz, Senior Member, IEEE, Javier Gómez-Aleixandre, Member, IEEE, and José Coto
}

\begin{abstract}
We propose an algorithm capable of solving the load power flow problem in ac droop-regulated microgrids. Based on radial distribution networks, these systems lack a slack bus for facilitating the computation by means of conventional methods. Rather than having the stiff bus that provides a voltage reference and supplies the necessary power, the voltage and power regulation must be shared among the distributed resources as a function of their frequency and voltage droop functions. The proposed algorithm is based on the well-known backward/forward sweep algorithm, conventionally employed to solve grid-connected radial load power flows, with the interesting property that they are derivative-free. We have expanded the algorithm to cope with the lack of slack bus. In this paper, we show the theoretical foundation and provide some tests with ex-post computations to investigate the coherence of the results.
\end{abstract}

Index Terms-Backward/forward sweep (BFS), droop, load power flow, microgrids.

\section{INTRODUCTION}

$\mathbf{T}$ HE SMART grid is a general term coined to describe an upgraded power system taking maximum advantage of information and communication technologies to accommodate new generation paradigms, and improve reliability and efficiency. The components of a smart grid are various and are rapidly innovating to accomplish these objectives, that the modern grid - with its load-oriented and centralized generation paradigms - might not meet in the future [1].

Precisely, microgrids are one of those smart grid components [1], which can ensure high reliability in view of their off-grid capabilities. In this sense, the use of droop regulation to provide the adequate stability to islanded microgridsoff-grid clusters of distributed generation, loads, and storage, on the basis of a distribution network-is currently a widely accepted communicationless paradigm. This is evidenced, for instance, in the reviews appearing in [2]-[4] or through the experimental support provided by demonstrative projects [5]. The droop regulation method is relatively simple, consisting in relating two variables of interest by means of a droop function.

Manuscript received December 12, 2014; revised April 9, 2015 and July 21, 2015; accepted September 3, 2015. This work was supported by the Spanish Ministry of Science under Grant ENE2010-14899. Paper no. TSG-01218-2014.

The authors are with the University of Oviedo, Gijón 33204, Spain (e-mail: guzman@uniovi.es).

Color versions of one or more of the figures in this paper are available online at http://ieeexplore.ieee.org.

Digital Object Identifier 10.1109/TSG.2015.2478278
Though several variants appear in the literature, most of the times the frequency and the real power generated are related by means of a constant frequency droop coefficient, $m_{P}$, following the function $\Delta f=m_{P} \Delta P_{G}$, which entails that any variation of the common frequency in the grid will provoke a proportional variation of the power supplied by each generator [2]. Similarly, usually we have $\Delta U=m_{Q} \Delta Q$, meaning that a variation of the bus voltage would provoke a variation in the generator reactive power, with an intensity defined by the voltage droop coefficient, $m_{Q}$. This procedure ensures that the burden of regulating the real and reactive powers is shared by all the generators, in inverse proportion to their droop coefficients, and without communication links. And it is this simplicity that seems to ensure the success of this paradigm; eventually considering it as the basic, primary level of hierarchical, more ambitious paradigms in which technical and economical layers have been proposed [2], [6].

In order to keep advancing in the definition of more complex paradigms, such as those analyzed in [2] and [6], it is necessary to adequately incorporate into the analysis all the factors that affect the microgrid performance. One of these factors is the grid topology, as it was recognized by Abdelaziz et al. [7]-[9], who in a series of recent papers succeeded in developing tools for analyzing the optimal performance of the microgrid. These authors recognized the advantages and opportunities of adapting a load power flow algorithm to the core of their approaches. This would eventually make it possible an expansion of the research possibilities, in parallel with the grid-connected distribution systems. However, these same authors also reported in [7] that the literature on algorithms for solving load power flow problems of droop-regulated microgrids was recent and scarce.

When the microgrid is tied to the main grid, the solution to the load power flow problem can be easily found by employing well documented methods. These have been divided in two main categories: 1) those requiring derivatives (Newton-Raphson, fast decoupled load flow, and similar); and 2) those that are not built upon Jacobian definitions, but only use basic circuit laws [backward/forward sweep (BFS) methods and others based on node equivalents] [10], [11].

The derivative-based methods are by large a translation of methods originally developed for the analysis of large, meshed power systems. They have been applied to the droopregulated microgrids in [12] and [13]. However, as it has been already detailed in [7], their approach was to define slack, 
$\mathrm{PQ}$, and PV buses, as in large power systems, disregarding the impact of the droop functions-particularly because their focus was on the electronic converter analysis. Based on this claim, Abdelaziz et al. [7] proposed an alternative Newtontrust region method, which they demonstrated to be efficient to account for the droop regulation. This they did by recognizing the difficulty of applying the BFS methods in an untied grid, because of the characteristic definition of such methods.

The derivative-free methods are a later introduction to cope with problems in the Jacobian-based methods. These problems have been revealed in a number of papers, starting with the seminal work of Shirmohammadi et al. [14]; where the authors complained that the Jacobian-based methods were not appropriate to solve practical problems presented when analyzing distribution networks (the reason behind the failure of Jacobian-based methods to give a proper solution can be traced back to the degradation of the predominance of the Jacobian diagonal, because of the comparatively large $\mathrm{R} / \mathrm{X}$ ratios of the distribution networks, which eventually makes the matrix prone to singularity [15]). Because of this, Shirmohammadi et al. [14] proposed using a BFS algorithm to solve the problem. This algorithm is based on the imperative condition that the network be radial, as it is the case of most distribution systems, in which though replication of current paths is possible, usually the topology is radial to limit the shortcircuit power and reduce the protection complexity [16]. The rationale is quite simple: it starts backwards (from the farthest buses) finding the line currents up to the reference bus (which may be the substation) assuming the voltages known, then it proceeds forward using the computed currents to update the voltages. There are variations in this general procedure, mainly in the way that the backward sweep is calculated from the different bus load models [17] or from using power rather than current in the branches [18]. But notwithstanding these minor adaptations, the main feature of the procedure is that it is generally robust and fast, as the reported rates of convergence compared to those of Newton-Raphson methods demonstrate in [11] and [19].

The solution that we propose herein is based on the BFS algorithms. That is, we have followed the alternative path to the derivative-based method in [7]. As in their case, the challenge in applying this approach is that we lack a stiff bus, which in a grid-connected microgrid is responsible for: 1) providing the necessary power for feeding the loads and satisfying the network losses; and 2) fixing a reference voltage for the BFS algorithm proper. In the islanded droop-regulated microgrid, the power is shared by a number of generators-in a proportion that depends on its droop function-and there is not a stiff voltage, but rather a compromise between generators to provide the equilibrium reactive power while accepting voltage variations in all the buses. Our approach remains simple, however. It is but an expansion of the BFS methods, in which upon the switch from backward to forward sweeps, we define the new voltage and frequency settings, which are communicated to all the buses over the subsequent forward sweep. This in turn will serve to update the complex power production of the distributed generators at the beginning of each backward sweep. The process is simple, and as in [20] we have implemented it in matrix form by employing the node incidence matrix as the process cornerstone, to avoid long iteration loops. The algorithm is devised entirely on matrix computations, even at the stage of updating the voltage at each individual node responding to the non-nominal frequency and voltage set-points issued by the droop-regulated generators. This simple and compact formulation avoids progressive sweeps that would otherwise reduce the performance.

Summing up, the main contributions of this paper are as follows.

1) As far as we know, this paper presents the first derivative-free algorithm to solve load power flows in islanded microgrids.

2) The proposed methodology overcomes the limitation of conventional BFS algorithms - namely, the need for a slack bus to serve as end and beginning of the backward and forward sweeps, respectively. The proposed algorithm distributes this feature among the intervening generators upon sweep changes in networks where there is not a slack bus.

3) This paper investigates the effect of inner and outer loop designs. It is discussed that the voltage updating design is a key issue in ensuring the convergence. The problems ensuing from conflicting updates are avoided by providing an outer loop with global voltage correction, which overlaps the individual voltage calculations.

4) Finally, this paper proposes a simple closed form formulation for defining the starting values, which is shown to clearly improve convergence features of the algorithm.

In what follows, we provide the theoretical foundation and the algorithm pseudo-code in Section II. Next in Section III we discuss the results obtained from a 33-bus test system, offering ex-post calculations to assess the coherence of those results. Section IV concludes this paper.

\section{Algorithm}

\section{A. Inner Loop: Frequency Droop}

1) Backward Sweep: The algorithm starts with a backward sweep, where the voltage nodes are fixed and assumed to be known. In our approach, also the deviation of the frequency from its nominal value, $\Delta f$, is assumed to be known by this stage. The first step of this backward sweep consists then in computing the required generation at each droop-regulated generator by acknowledging the common frequency signal. That is

$$
\Delta P_{G i}=\frac{\Delta f}{m_{P i}}, \quad i=2, \ldots, N .
$$

(In our implementation, we have considered that each node is endowed with a droop-regulated generator. We employ this strategy to speed up the computation by employing vector notation. If this is not the case and $\mathcal{G}$ is the index set of those nodes that do have attached generation, we identify the nongenerating nodes by simply stating at the preliminary declarations, before running the algorithm, that $m_{P i} \rightarrow \infty$ if $i \notin \mathcal{G}$.)

In (1), we define the droop by means of deviations from a nominal value that we denote by the superscript 0 : $\Delta P_{G i}=P_{G i}-P_{G i}^{0}$ and $\Delta f=f-f^{0}$. Also note importantly 
that the computation of $\Delta P_{G i}$ in (1) is conducted for all nodes except for the arbitrarily chosen as reference node, which we also have labeled as node number 1 . This node 1 will later act as a substation node similar to that in a grid-connected distribution system. But in this case, the frequency and voltage of this node will be floating to accommodate the requisites of load balance.

At each node, we compute the drawn power as

$$
\begin{aligned}
S_{i}= & \left(P_{L i}+P_{G i}\right)+j\left(Q_{L i}+Q_{G i}\right) \\
= & \left(P_{L i}+P_{G i}^{0}+\Delta P_{G i}\right) \\
& +j\left(Q_{L i}+Q_{G i}^{0}+\Delta Q_{G i}\right), \quad i=2, \ldots, N .
\end{aligned}
$$

Once known the power drawn from each nonreference bus, the current can be computed from

$$
I_{i}=\frac{S_{i}}{U_{i}}, \quad i=2, \ldots, N .
$$

The backward sweep then proceeds by computing all the branch currents starting from the farthest nodes and proceeding upstream

$$
I_{i j}=I_{j}+\sum_{m \in \mathcal{A}_{j}} I_{j m}, \quad j=N, \ldots, 2
$$

where $\mathcal{A}_{j}$ is the index set of the nodes that are adjacent to $j$.

This procedure is, however, quite inefficient. It requires a progressive sweeping of the nodes, which involves the investigation of connections and the use of loops. We therefore have opted by a direct approach in which the values of the branch currents are found in one step by means of matrix computation; particularly using the node incidence matrix.

In general, the node incidence matrix $\boldsymbol{\Gamma}$ is an $N_{b} \times N$ matrix, where $N_{b}$ is the number of branches, such that its element $\gamma_{i j}$ is [21], [22].

1) $\gamma_{k l}=+1$ when current in branch $k$ leaves node $l$.

2) $\gamma_{k l}=-1$ when current in branch $k$ flows toward node $l$.

3) $\gamma_{k l}=0$ when no connection exists.

According to network theory, branch and bus current vectors following the ordering in the definition of $\boldsymbol{\Gamma}$ are linked as follows:

$$
\mathbf{I}_{\text {node }}=\Gamma^{t} \mathbf{I}_{\text {branch }}
$$

where the superscript $t$ indicates transpose.

This means that from the branch currents we can easily obtain the bus currents once the topological structure of the distribution system is known. However, in the backward sweep we are interested in computing the branch currents from the bus injected currents. Therefore, we can summarize (4) in

$$
\mathbf{I}_{\text {branch }}=\boldsymbol{\Gamma}^{-t} \mathbf{I}_{\text {node }}
$$

where the superscript $-t$ indicates inverse transpose.

It can be argued that in general $N_{b} \neq N$, and hence $\boldsymbol{\Gamma}$ is not generally invertible, making impossible the step from (5) to (6). However, in a purely radial systems it happens that $N_{b}=N-1$. So by eliminating from $\Gamma$ the first column, corresponding to the reference node, we can directly compute the branch currents from the node currents in just one matrix operation. (We can eliminate the column of $\boldsymbol{\Gamma}$ representing the first, reference node because indeed this node is not part of the backward process. This process ends at the adjacent nodes of the reference node. Node number 1 is exclusively employed at the beginning of the forward sweeps as the responsible of establishing the frequency and voltage deviations derived from its generator droop functions.)

This proposal for directly computing the branch currents in just one step agrees with the procedure investigated in [20], when he exploited the use of a bus injection to branch current, BIBC, matrix to link bus injected currents to branch currents. An analysis of the specific procedure to build BIBC on the basis of the topological structure indicates nonetheless that $\mathbf{B I B C}=\boldsymbol{\Gamma}^{-t}$, and that the two matrix descriptions are equivalent for computing the "undrooped" power flow.

2) Forward Sweep: In the conventional BFS method, the reference node is employed as the base for progressing downstream correcting the bus voltages now that the branch currents are known

$$
U_{j}=U_{i}-Z_{i j} I_{i j}, \quad j=2, \ldots, N .
$$

To this purpose, the voltage of node 1 is considered fixed (usually equal to 1 p.u.), hence being assumed to be a slack bus in the conventional power system analysis framework. The algorithm uses this $U_{1}$ voltage as the reference voltage to compute the rest of the bus voltages.

Additionally, we also employ this node 1 to redefine the frequency of the microgrid. After finishing the backward sweep, the current (power) of the branch leaving the node number 1 is expected to be in general not zero. Particularly, in a setting where the node is the substation feeding the distribution system, it is assumed that this branch current is drawn from or injected into this node, depending on whether there is a shortage or an excess of generation in the distribution system. The reference node is therefore the gateway to the bulk power system, through which a power $S_{1}=\sum_{i \in \mathcal{A}_{1}} U_{1} I_{1 i}^{*}$, is exchanged. In our setting, however, we can employ this traded power to indicate the frequency in the microgrid. We know that the following equilibrium must hold in node 1 :

$$
P_{G 1}+P_{L 1}+\operatorname{Re}\left(\sum_{m \in \mathcal{A}_{1}} U_{1} I_{1 m}^{*}\right)=0 .
$$

Also, $P_{G 1}=\Delta P_{G 1}+P_{G 1}^{0}$. So we can employ the branch currents ensuing from the backward sweep to determine the new frequency in the entire microgrid

$$
\Delta f=-m_{P 1}\left[P_{L 1}+P_{G 1}^{0}+\operatorname{Re}\left(\sum_{i \in \mathcal{A}_{1}} U_{1} I_{1 i}^{*}\right)\right] .
$$

This new $\Delta f$ is stored and will be later employed in (1) to proceed with the next backward sweep. The deviations in the frequency will be the signal in the backward sweep to curtail or increase the generating power. But for the forward sweep it is not required anymore. The forward sweep proceeds efficiently in matrix form by computing the error in the previously 
assumed known voltages. Graph theory shows that (as before, for the special case of a square incidence matrix)

$$
\mathbf{U}_{\text {node }}=\Gamma^{-1} \mathbf{U}_{\text {branch. }}
$$

In our case, $\mathbf{U}_{\text {branch }}$ is the voltage drop in the grid lines, which can be obtained as

$$
\mathbf{U}_{\text {branch }}=\operatorname{diag}\left(\mathbf{Z}_{\text {branch }}\right) \mathbf{I}_{\text {branch }}
$$

where $\operatorname{diag}\left(\mathbf{Z}_{\text {branch }}\right)$ is the diagonal matrix of ordered line impedances. Accordingly, the increase of the bus voltages in the forward sweep is obtained through

$$
\Delta \mathbf{U}=\boldsymbol{\Gamma}^{-1} \operatorname{diag}\left(\mathbf{Z}_{\text {branch }}\right) \mathbf{I}_{\text {branch }}
$$

and the updated voltages through

$$
\mathbf{U}=\mathbf{U}^{0}-\Delta \mathbf{U}
$$

where $\mathbf{U}^{0}$ is a vector with all entries equal to the initial assumed values; usually 1.0 p.u.

Again this direct procedure is equivalent to that presented in [20] for grid-connected systems, where Teng proposed the use of a branch current to bus voltage matrix, $\mathbf{B C B V}$, to directly solve the bus voltages updating. Just by examination, it can be found that $\mathbf{B C B V}=\boldsymbol{\Gamma}^{-1} \operatorname{diag}\left(\mathbf{Z}_{\text {branch }}\right)$ (further analysis can be found in [19]).

\section{B. Outer Loop: Voltage Droop}

The previous algorithm finds the power flow in the microgrid-along with the frequency setting - by assuming that the voltage of the reference node is constant and equal to a predefined value $\mathbf{U}^{0}$. However, the need for reactive power sharing through voltage droops of the type $m_{Q i}=$ $\left(\Delta U_{i} / \Delta Q_{G i}\right)$ makes this assumption not valid. If the reference generator is endowed with a voltage droop control, its voltage should depart from the initial $U_{1}^{0}=1.0$ p.u. once that the reactive power in the microgrid is modified.

As a solution to the updating of $\mathbf{U}^{0}$ according to the reactive droop gains, we first devised an update at each conventional sweep. Let that sweep be called the inner loop, which is responsible for computing, in its forward sweep, the bus voltages. The update is presented as $\Delta \mathbf{U}$. Proceeding at the same time with the reactive sharing calculations gave us convergence problems, because sometimes conflicting updates where issued by the voltage. Particularly depending on the initial guess of reactive power generation and bus voltages, the forward sweep might ask for a negative increase of bus voltages at the next iteration, whereas the droop voltage function might ask for a positive increase. In our experience this occurred in a substantial number of instances when the reactive power initial guess was not carefully selected. As a result, the algorithm oscillated or diverged.

As an alternative solution, we introduced the voltage droop in an outer loop. With a procedure similar to that of the frequency droop, the outer loop computes $\Delta Q_{G i}$ in the backward sweep, and employs the droop of the reference generator to set the voltage deviations, $\Delta U_{i}^{\prime}$, in the forward sweep. These deviations of reactive power and voltage are introduced into the inner loop, which is in charge of giving the corresponding power flow. In other words, the inner algorithm finds the equilibrium of the microgrid-frequency droop functions included-every time that the outer algorithm forces the reactive powers and voltages to change.

The procedure is thus similar to that of the frequency droop; but not equal. Whereas the frequency is the same for all the microgrid, the voltage is not, which is a problem because it seems at first that we should iterate over all the existing buses to get the correct equilibrium between both voltage and reactive power; which are related through each individual droop function. However, if we examine (13) we observe that the voltage updates in the inner loop are the result of modifying the initially predefined values $\mathbf{U}^{0}$ by an amount given by $\Delta \mathbf{U}$. Indeed, this is an ingenious way of avoiding the loop computation shown in (7). Rather than starting at node 1 and proceeding downstream updating the node voltages, the deviation is found in just one step; i.e., (12). This is advantageous, because it means that the whole system voltages can be obtained from a reference voltage at the reference node in just one matrix operation. Therefore, if we employ this inner-outer loop strategy, we see that we can indeed change the value of the reference voltage at each outer iteration and let the inner loop proceed with the computation of the node voltages.

More precisely, after the inner loop ends, we define the voltage error at node 1 , similarly to (9), as

$$
\Delta U_{1}^{\prime}=-m_{Q 1}\left[Q_{L 1}+Q_{G 1}^{0}+\operatorname{Im}\left(\sum_{i \in \mathcal{A}_{1}} U_{1} I_{1 i}^{*}\right)\right] .
$$

This is the update of the voltage of the reference bus. Therefore, we can hereafter proceed with the inner loop after informing it that the "slack" bus has a new voltage that is $U_{1}^{0}-\Delta U_{1}^{\prime}$.

\section{Summary}

The algorithm is described next, with both inner and outer loops featured in the following pseudocode.

$$
\begin{array}{lr}
\mathbf{U}^{0^{\prime}} \leftarrow \mathbf{U}^{0} & \\
\text { while } \Delta U_{1}^{\prime} \neq \text { const. do } & \triangleright \text { outer loop } \\
\Delta Q_{G i} \leftarrow \frac{\Delta U_{1}^{\prime}}{m_{Q i}}, \quad i \neq 1 & \\
\Delta f \leftarrow 0 & \\
\Delta \mathbf{U} \leftarrow \mathbf{0} & \\
\text { while } \Delta f \& \Delta \mathbf{U} \neq \text { const. do } & \\
\Delta P_{G i} \leftarrow \frac{\Delta f}{m_{P i}}, \quad i \neq 1 & \triangleright \text { BS starts } \\
\text { Compute } S_{i}, \quad i \neq 1 & \triangleright \text { Eq. (2) } \\
\text { Compute } \mathbf{I}_{\text {node }}, \text { then } \mathbf{I}_{\text {branch }} & \\
\Delta f \leftarrow-m_{P 1} \times P_{1} & \triangleright \text { FS starts, Eq. (9) } \\
\text { Compute } \Delta \mathbf{U} & \triangleright \text { Eq. (12) } \\
\mathbf{U} \leftarrow\left(\mathbf{U}^{0^{\prime}}-\Delta \mathbf{U}\right) & \\
\text { end while } & \\
\Delta U_{1}^{\prime} \leftarrow-m_{Q 1} \times Q_{1} & \\
\mathbf{U}^{0^{\prime}} \leftarrow \mathbf{U}^{0}-\Delta U_{1}^{\prime} &
\end{array}
$$




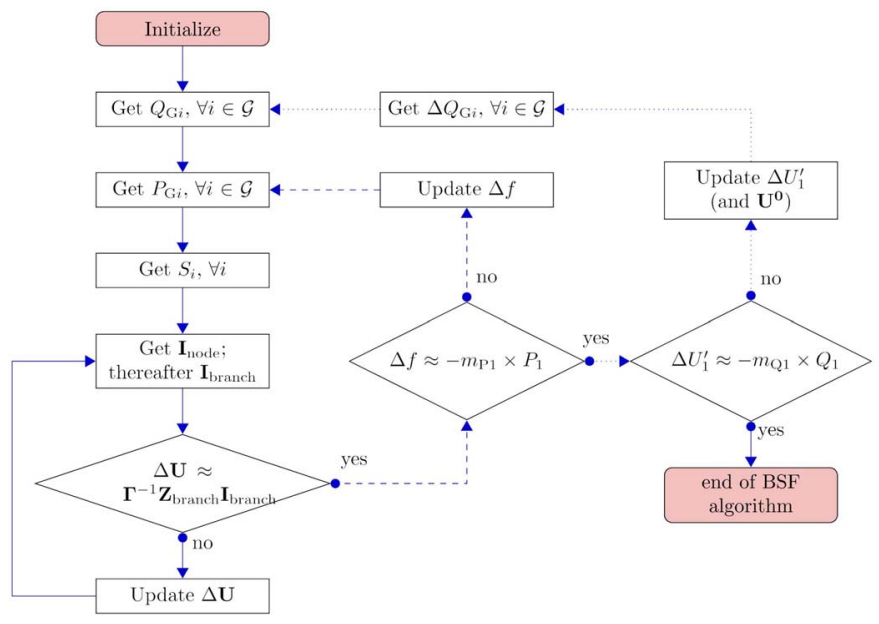

Fig. 1. Algorithm flow chart. Dashed lines show the inner (frequency) loop, and dotted lines show the outer (voltage) loop.

Also, a flow chart is shown in Fig. 1. Note that in the flow chart a variation of the proposed algorithm is shown. The updating of $\Delta f$ is made after each stabilization of $\Delta \mathbf{U}$; rather than searching the convergence of both variables at the same time. This provides an alternative programming procedure. Nonetheless, in our tests this alternatives did not demonstrate significant differences in speed or convergence. (Not so as discussed above, the simultaneous updating of $\Delta U_{1}^{\prime}$ and $\Delta \mathbf{U}$, which showed remarkable problems of stability in the convergence.)

\section{DISCUSSION}

\section{A. Numerical Results}

In what follows, we discuss some numerical results regarding the use of the proposed algorithm, evidencing by means of ex-post calculations that the equilibrium points found by the algorithm are coherent. The benchmark that we have employed is the 33-bus test system described in [23], which we have plotted in Fig. 2. The original test system consisted of a two feeder substation, 32 buses, and five looping branches (tie lines), with a base voltage of $12.66 \mathrm{kV}$. (Additionally we have employed a base power equal to $500 \mathrm{~kW}$.) Originally in [23] the system was tied to the bulk power system through node number 1 .

1) Validation of the Grid-Connected Microgrid Results: In Fig. 2, we present the results of a preliminary simulation of the test system, with the microgrid connected to the main grid and without any distributed resource. That is, we provide the same base case as in [23] with results similar to that reported elsewhere in the literature (see for instance [24]). We obtained these results by setting all the droop coefficients of the nonreference bus to infinity and their nominal powers to zero, meaning that the supplied power would have been zero regardless of any variation in the frequency or node voltages. (We set the nominal powers to zero so that the system is exactly the same as in [23]. This zero generated power is not a prerequisite in a general case, however, because any generated power in the $i$ th generator may be selected just by setting $P_{G i}^{0}$ to a negative value equal to the desired generated power.)
In the reference bus we set $m_{P 1}=m_{Q 1}=0$, meaning that the reference bus would be capable of altering its power-as much as the load required-with a constant $\Delta f=\Delta U_{1}^{\prime}=0$.

2) Coherence of the Solution for the Islanded Microgrid Problem: The results presented in Fig. 3 concern to the islanded microgrid employing the same grid. We again used the node 1 as a reference bus, but in this occasion the setting included droop regulation in five buses, including node number 1. In Fig. 2, the slack bus injected a complex power equal to $-7.836-j 4.871$ p.u., which compensated the load power $(7.430+j 4.600$ p.u. $)$ plus the line consumptions $(0.405+j 0.270$ p.u. $)$. In the islanded systems, we distributed the generated power equally among the five generators by setting their nominal power equal to $0.9+j 0.9$ p.u. This left a residual power of $3.336+j 0.371$ p.u. that the algorithm should compensate by modifying the values $\Delta f$ and $\Delta U_{1}^{\prime}=0$. Eventually this modification would be dictated by the droop coefficients, which we defined as $\left(m_{P 1}, m_{P 6}, m_{P 13}, m_{P 25}, m_{P 33}\right)=$ $(-0.05,-1.00,-0.10,-1.00,-0.20)$. For simplicity in the analysis, at each bus we set $m_{P i}=m_{Q i}$.

After running the algorithm, we obtained the new equilibrium points. The (inner loop of the) algorithm found that the frequency deviation in this microgrid is 0.081 p.u., which can be demonstrated to agree with a rough estimate by hand.

1) First, we can state that the "stiffness" of the whole microgrid is given by an equivalent droop coefficient $m_{P T}$ that can be calculated as

$$
m_{P T}=\left(\sum_{i \in \mathcal{G}} m_{P i}^{-1}\right)^{-1}=-0.027 .
$$

2) Next, the original real power drawn from the main grid (see Fig. 2) was -7.836 p.u., which now should be undertaken by the five distributed resources.

3) The equivalent nominal power of the microgrid represented in Fig. 3 is $P_{\mathrm{GT}}^{0}=5 \times 0.9=4.5$ p.u. This entails a mismatch of the real power equal to $\Delta P_{\mathrm{GT}}=-7.836+4.5=-3.336$ p.u.

4) It follows then from the droop function that the frequency deviation should be $\Delta f=m_{\mathrm{PT}} \Delta P_{\mathrm{GT}}=$ 0.09 p.u., which is quite close to the final value of 0.081 p.u.

The difference between the estimate by hand and the exact computation by the algorithm resides in the line losses and the actual generated power. The total load in this network configuration was the same as in Fig. 2. The losses were remarkably lower, however, as expected from the distribution of generation: $0.035+j 0.028$ p.u. Had we known the generation and losses beforehand-which we obviously did not-we would have known that $\Delta P_{\mathrm{GT}}=-7.465-0.035+4.5=-3.0$ p.u. was the required deviation of the power from its nominal value. Hence we would have corroborated that $\Delta f=-0.027 \times 3.0=$ 0.081 p.u.

Similar conclusions can be inferred from the deviation of voltage $\Delta U_{1}^{\prime}=0.003$ p.u. By the way the algorithm is constructed, this deviation is spread throughout the microgrid. It is 


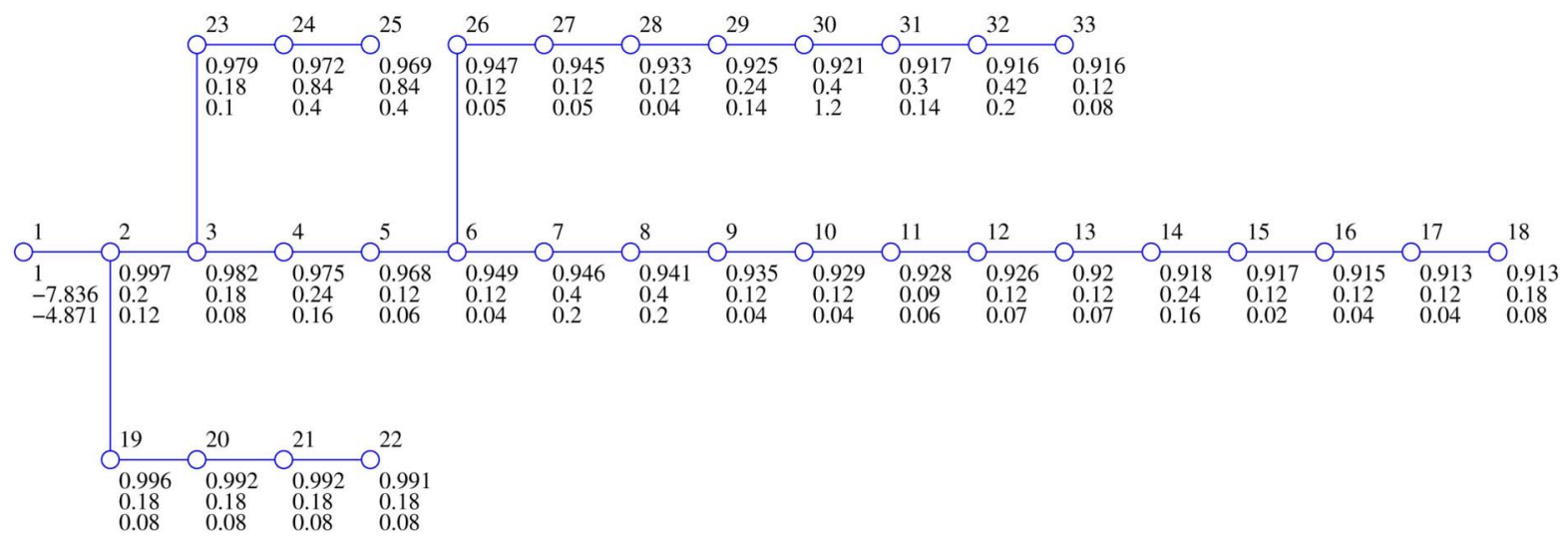

Fig. 2. 33-bus test system. Base case of the grid-connected microgrid. Node 1 is the slack bus. For each node voltage, real power, and reactive power (from top to bottom) are provided in p.u. Bases are $12.66 \mathrm{kV}$ and $500 \mathrm{kVA}$.

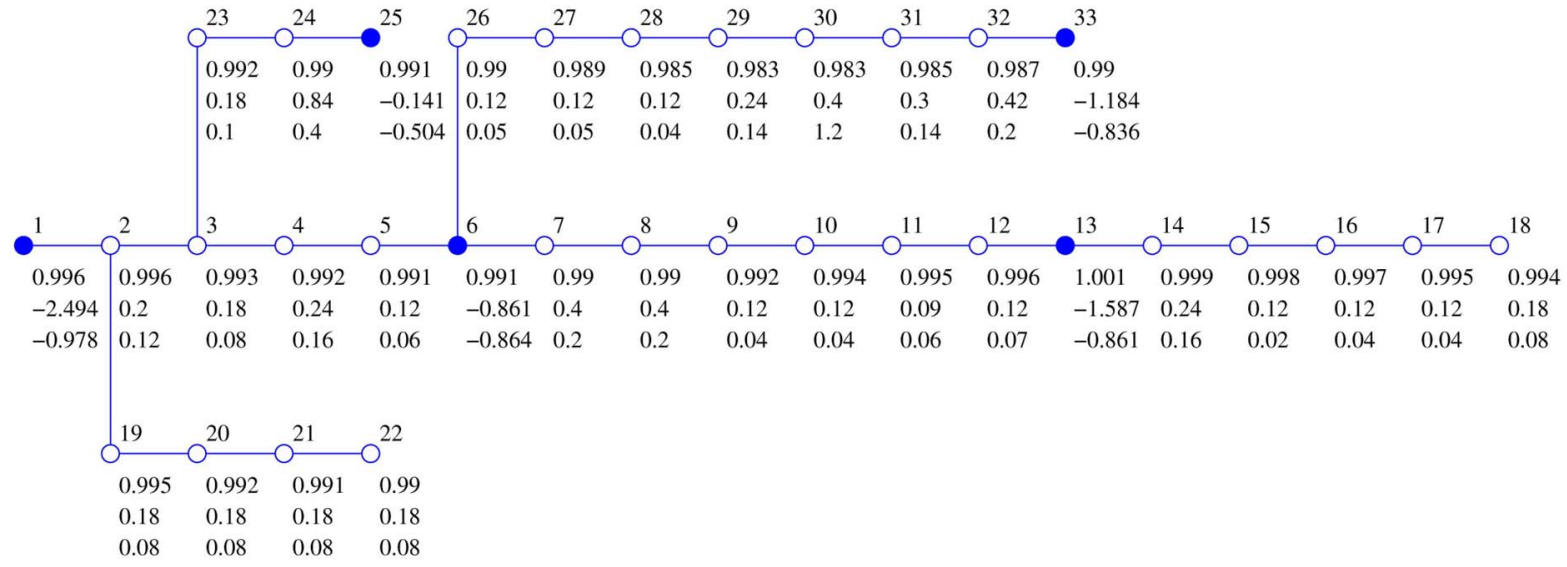

Fig. 3. Islanded microgrid. Droop-regulated generators are denoted by bold circles.

only readily visible in node 1 , however, because it is the reference node in the algorithm, and therefore it is affected only by the correction in voltage imposed by the outer loop of the algorithm. The remaining node voltages follow the drop imposed by the reference node, but at the same time they are affected by further corrections made by the inner loop. In this sense, it is interesting to note that not only the reference node has lost its stiffness-being forced to have a voltage below 1.0 p.u., which is a clear evidence of the islanded condition-but also that node number 13 has now a voltage greater than that of the reference node.

3) One Only Droop-Regulated Generator: The previous example shows that the reference node does not differ in its behavior from other generator buses. Indeed, if we select node 25 as the reference node, the result would have been the same. Also, the algorithm can solve the network with just one generator bus, which would obviously be the reference node. Fig. 2 was an instance of such a case in which the generator settings were $m_{P 1}=m_{Q 1}=0$. But we may change those settings to the values employed in Fig. 3 and eliminate the other four generators, and the algorithm would find the correct solution. Particularly, the result would be $\Delta f=0.362$ p.u. and $\Delta U_{1}^{\prime}=0.209$ p.u., so the reference node 1 would deplete its voltage according to the voltage droop down to 0.791 p.u., in coherence with the reactive power demand and line consumption equal to $4.6+0.479=$ 5.079 p.u. $\left(\Delta Q_{G 1}=-5.079+0.9=-4.179\right.$ p.u., and hence $\Delta U_{1}^{\prime}=-0.05 \times-4.179=0.209$ p.u. $)$.

4) Coherence of the Load Sharing: Also the load is distributed by the algorithm correctly among the generators in accordance with their respective droops. This is demonstrated with the help of Fig. 4, where as way of comparison the demand at the bus 18 is increased threefold. We did not change the droop coefficients from the values of Fig. 3. The increase of the load in bus 18, plus the new distribution of losses, are again shared by all the generators. However, from the previous scenario the load supplied by node 13 has increased by $\Delta P_{13}=1.675-1.587=0.088$ p.u., whereas at bus 6 the increase has been only $\Delta P_{6}=0.87-0.861=0.009$ p.u. (Here we point out that these powers are not the power generated by the distributed resources. To obtain it, we must subtract the power drawn by the load, which can be accessed from Fig. 2. Obviously, this does not affect the analysis in this paragraph, because we are dealing with increments.) If we revise the frequency droop coefficients, we observe that the result provided by the algorithm is again correct, because $\left(m_{P 13} / m_{P 6}\right)=(0.1 / 1.0) \approx\left(\Delta P_{6} / \Delta P_{13}\right)$. And it is not that the generator bus number 13 is closer to the bus that increases 


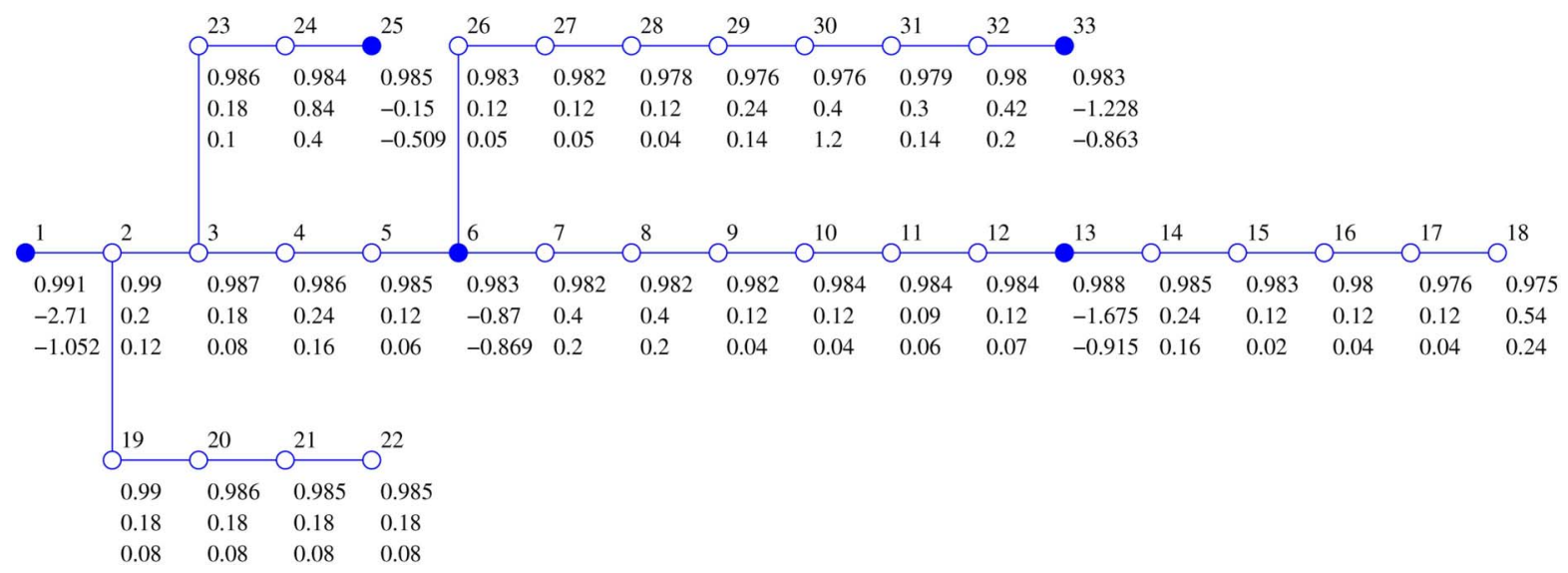

Fig. 4. Same as Fig. 3, but with node 18 three times more loaded.

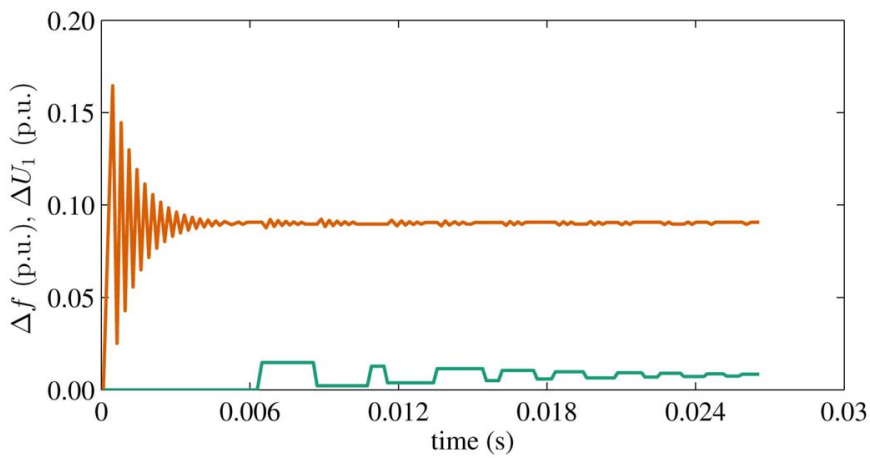

Fig. 5. Convergence of the solution of the test shown in Fig. 3. The top (red) line represents $\Delta f$ and the bottom (green) line is $\Delta U_{1}^{\prime}$.

its load. If we repeat the test, but instead similarly increasing the load of bus 22 , which has the same load in the original grid as bus 18 , again the results repeat: $P_{6}=-0.87$ and $P_{13}=-1.676$ p.u. Only a slight difference in the results can be observed, due to the reallocation of grid losses. Also, the same attribution of reactive power as a function of the inverse voltage droop coefficients can be observed, implying that the computations made by the outer loop of the algorithm also give coherent results.

\section{B. Convergence Process}

Fig. 5 evidences the process of convergence of the algorithm on the basis of its two nested loops. The algorithm employs the deviation of the frequency and voltages as stopping criteria. In this simulation, we defined the initial guess values of the droop-related deviations as $\Delta f=\Delta U_{1}^{\prime}=0$. We also set the deviations of the bus voltages to zero; i.e., $\Delta \mathbf{U}=0$. With these settings, the algorithm proceeds to find the solution after running 18 iterations of the outer loop, as shown by the bottom (green) line of Fig. 5. Each time that the reference voltage is corrected-entailing a new distribution of reactive power-the inner loop proceeds to find the new value of $\Delta f$ and hence the new distribution of real power among the generators (top, red line). It is important to note that the value of $\Delta f$ must not be reset when $\Delta U_{1}^{\prime}$ is corrected. If we let the inner loop start from the last "remembered" $\Delta f$, the large oscillations

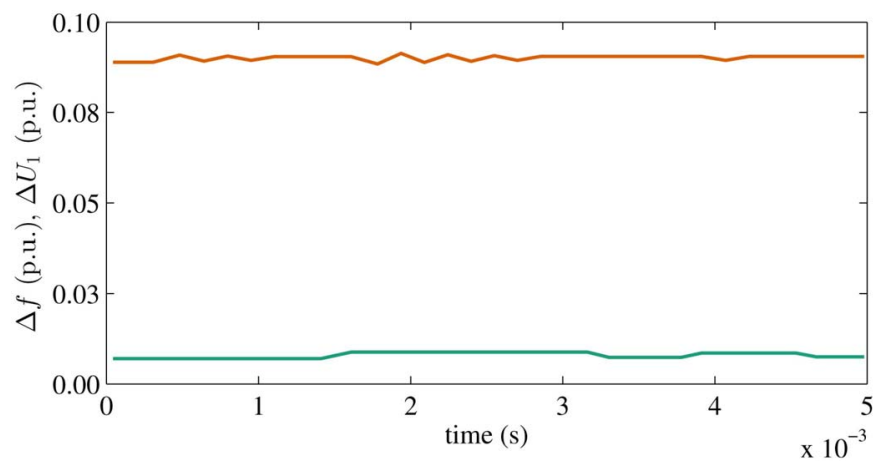

Fig. 6. Same case as in Fig. 5, but with initial guesses following (16) and (17): $\Delta f=0.089$ p.u. and $\Delta U_{1}^{\prime}=0.007$ p.u.

observed during the first $6 \mathrm{~ms}$ are avoided, and a set of small oscillations ensues as it can be observed in the plot. In doing this, the convergence speed is appreciably increased, as it can be readily observed in Fig. 5, where the first stint to find the real power equilibrium is the longest.

Based on this observation, the convergence speed can be increased by providing good estimates that prevent the first set of oscillations. As we outlined above when we checked the validity of the results obtained from the use of the algorithm, we can obtain a reasonable good estimate if we neglect the power drawn by the line impedances and approximate the total generated power to be equal to the total demanded load. Particularly, we can approximate the initial value of the frequency deviation as follows:

$$
\Delta f=-\left(\sum_{i \in \mathcal{G}} m_{P i}^{-1}\right)^{-1}\left(\sum_{i=1}^{N} P_{L i}+\sum_{i \in \mathcal{G}} P_{G i}^{0}\right) .
$$

This approximation ensues from the assumption of null line losses as a first rough approximation. If those losses are neglected, all generators are in parallel, and hence an equivalent generator can substitute the entire generation by directly summing the required power and associating the droops in parallel. That is, the droop coefficient of an equivalent generator, $m_{\mathrm{PT}}$, would be in this way related to the individual droop coefficients as $\left(1 / m_{\mathrm{PT}}\right)=\sum_{i \in \mathcal{G}}\left(1 / m_{P i}\right)$. 


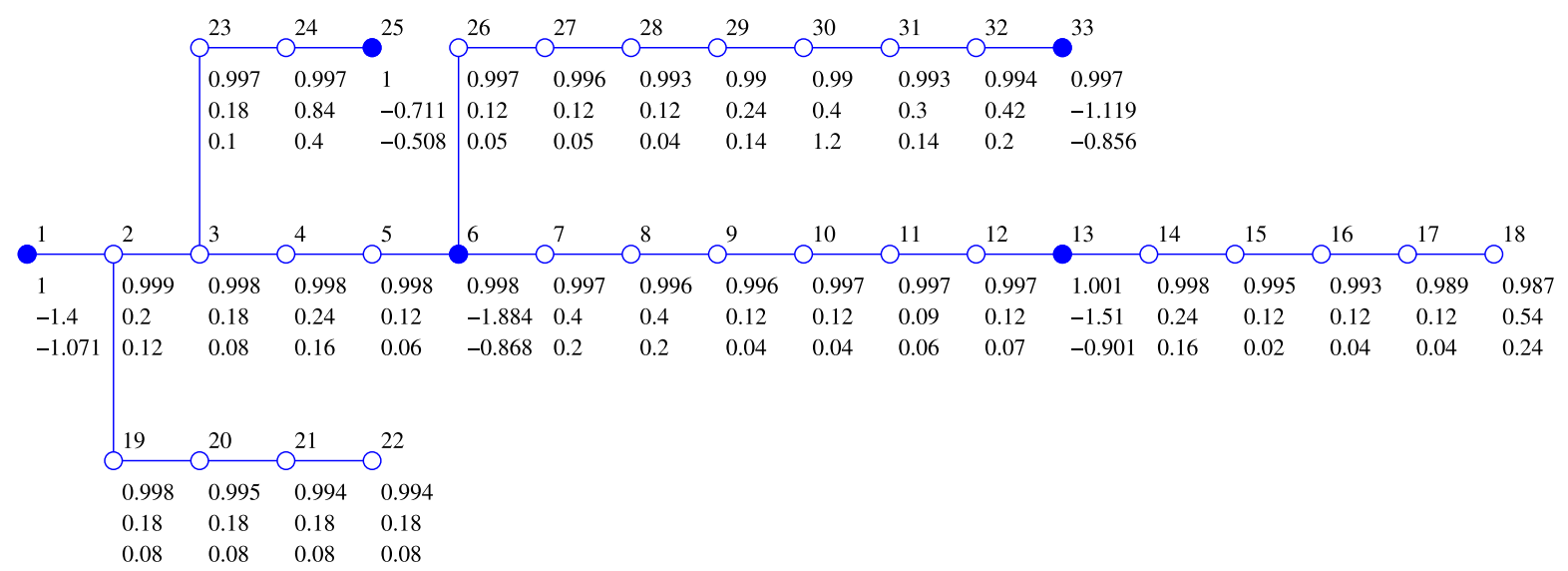

Fig. 7. OPF ensuing from the scheduling of droop coefficients. It should be compared to Fig. 4.

Similarly, a reasonable first estimate of the deviation of the reference node voltage can be obtained as

$$
\Delta U_{1}^{\prime}=-\left(\sum_{i \in \mathcal{G}} m_{Q i}^{-1}\right)^{-1}\left(\sum_{i=1}^{N} Q_{L i}+\sum_{i \in \mathcal{G}} Q_{G i}^{0}\right) .
$$

(Note that throughout this paper we have considered the numerical value of load power as positive, the generation power as negative, and the droop coefficient negative.)

As it can be observed in Fig. 6 the speed of convergence is notably improved, with fewer oscillations of the frequency loop and just five iterations of the outer loop. The tolerances were $10^{-6}$ and $10^{-3}$ p.u. for the voltage and frequency loops, respectively. The time shown in the horizontal axis is CPU time of a 64-bit laptop with processor Intel Core i7-4710HQ $2.50 \mathrm{GHz} 8.0 \mathrm{~GB}$ RAM.

Summing up, the major issues that we have detected regarding convergence are as follows.

1) The order in which $\Delta f$ (the frequency droop) and $\Delta \mathbf{U}$ are updated does not seem to be an issue from the speed and stability point of view.

2) The updating sequence does represent an important issue of convergence in the reactive power droop control. Simultaneous updating of the global and local voltages leads to instability in some scenarios.

3) Speed is affected by resetting the inner frequency loop once the outer loop is updated.

4) Finally, the speed is greatly increased and the convergence ensured by choosing reasonable initial guesses; such as the ones provided in (16) and (17).

\section{Extension to Optimization Problems}

The algorithm can be readily employed to formulate optimization problems in which the decision must be constrained by a valid power flow solution. As an illustration, here a simple example is proposed in which a kind of optimal power flow (OPF) is solved to find the microgrid configuration that entails minimum power losses.

The problem can be approached through a two-stage program in which the top-level program seeks to optimize the power losses by defining a set of decision variables, which

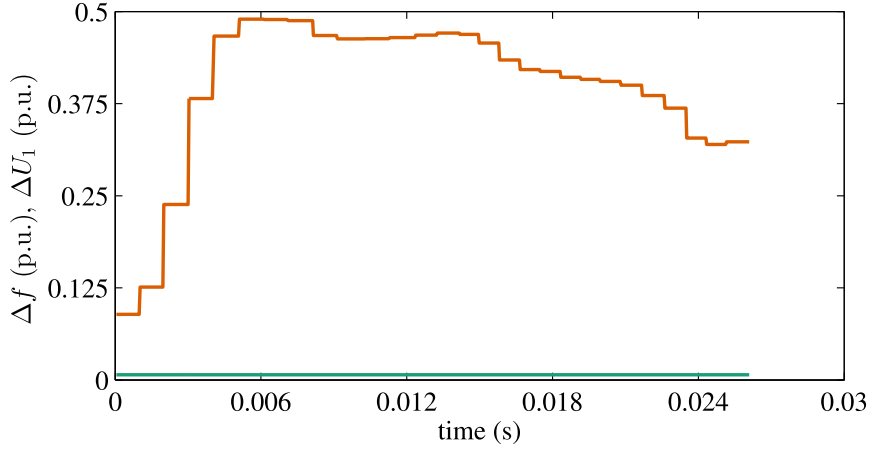

Fig. 8. Convergence of the solution to the illustrative OPF problem.

are fed to the low-level program in which the BFS algorithm is solved. In this particular example, we choose to find the droop scheduling - that is, the values of the droop coefficients (see [25]) - that once the nominal powers are defined minimize the losses. In other words, we selected the coefficients $m_{P i}, i \in \mathcal{G}$ as the decision variables.

The starting point for this example is the setup of Fig. 4. with droop coefficients of generators in nodes 1, 6, 13, 25 , and 33 equal to $(-0.05,-1.00,-0.10,-1.00,-0.20)$. The ensuing losses were 0.0403 p.u. We employed the optimization approach by defining an interior-point-based optimization algorithm in which the objective function, the grid losses, is computed at each iteration by solving the proposed BFS algorithm; with the provision that the decision variables were bounded between -1.1 and -0.04 (to avoid reaching an infeasible point because of bifurcation [25]). The algorithm finds the minimum losses to be 0.0314 p.u. when the ensuing droop coefficients are $(-0.6904,-0.2927$, $-0.4432,-0.4968,-0.9541)$. In the original, nonoptimal solution the generators that participated more prominently in the power supply were located at nodes 1 and 13. These were the generators with the lowest droop coefficients, which assumed most of the variation from the nominal point. After optimizing, more emphasis is given to the generator in node 6, which is more centered (Fig. 7). That is, its droop coefficient is the lowest $\left(m_{P 6}=-0.2927\right)$ and its power production the largest. 
The solution to this illustrative OPF problem shows that the frequency deviation is remarkable. This deviation can be obtained as a by-product of the solution of the BFS at each iteration step of the interior-point algorithm. Its evolution is shown in Fig. 8, where it is observed how it departs from the nonoptimal, original value 0.09 (see Fig. 6) to a final value of 0.32 . The step-wise evolution represents the iteration of the top-level interior-point algorithm, with the oscillations of the low-level algorithm obscured by the scale, because of the adoption of the approximated initial guesses, as in Fig. 6, at each top-level iteration. (Obviously, this large deviation in the frequency may be considered excessive. We did not mean to make a detailed analysis, but an illustration of how the BFS algorithm can be easily incorporated into a two-level optimization program. Expansion to account for bounds and constraints to avoid such results should be straightforward in the top-level program.)

\section{CONCLUSION}

We have proposed an algorithm for solving the load flow problem of droop-regulated islanded microgrids. The algorithm is a derivation of the BFS method widely used in the analysis of distribution networks connected to the main grid. In our approach, however, we have avoided the use of a slack bus with fixed voltage and infinite power capability. We propose a methodology based on updating the voltage and frequency deviations ensuing from the droop functions upon the switch from backward to forward sweep. This update is transferred to each node following the forward sweep, enabling the update of bus voltages and powers by employing the droop function of each individual generator. We have tested the approach in a 33-bus distribution system, and have provided evidence about the coherence of the ensuing results. Finally, we have commented how the speed of the algorithm can be boosted by a proper definition of the initial guess, which we have presented in closed form. On the whole, the algorithm inherits the properties of other grid-connected BFS methods, and with its specific characteristics it opens the way to a more complete analysis of islanded microgrids in which the network characteristics are considered.

The immediate application of the algorithm is to analyzing the primary equilibrium of an islanded microgrid in given conditions of load and droop settings. However, the algorithm may be readily adapted to optimization frameworks. The lack of gradient definitions makes it possible to straightforwardly explore the optimal droop settings-gains and nominal powers-under the subject of for instance OPF. As it is readily inferred from the structure of the input data, the values defining the regulated power can be promptly employed as decision variables of a major two-stage optimization problem in which the solution to the proposed algorithm remains at the second level. In all, the speed demonstrated by the algorithm shows promising application to such investigations.

\section{REFERENCES}

[1] P. P. Varaiya, F. F. Wu, and J. W. Bialek, "Smart operation of smart grid: Risk-limiting dispatch," Proc. IEEE, vol. 99, no. 1, pp. 40-57, Jan. 2011
[2] D. E. Olivares et al., "Trends in microgrid control," IEEE Trans. Smart Grid, vol. 5, no. 4, pp. 1905-1919, Jul. 2014.

[3] E. Planas, J. Andreu, J. I. Gárate, I. Martínez de Alegría, and E. Ibarra, "AC and DC technology in microgrids: A review," Renew. Sustain. Energy Rev., vol. 43, pp. 726-749, Mar. 2015.

[4] E. Planas, A. Gil-de-Muro, J. Andreu, I. Kortabarria, and I. Martínez de Alegría, "General aspects, hierarchical controls and droop methods in microgrids: A review," Renew. Sustain. Energy Rev., vol. 17, pp. 147-159, Jan. 2013.

[5] N. Lidula and A. Rajapakse, "Microgrids research: A review of experimental microgrids and test systems," Renew. Sustain. Energy Rev., vol. 15 , no. 1, pp. 186-202, Jan. 2011.

[6] J. M. Guerrero, J. C. Vasquez, J. Matas, L. G. de Vicuna, and M. Castilla, "Hierarchical control of droop-controlled AC and DC microgrids: A general approach toward standardization," IEEE Trans. Ind. Electron., vol. 58, no. 1, pp. 158-172, Jan. 2011.

[7] M. M. A. Abdelaziz, H. E. Farag, E. F. El-Saadany, and Y. A.-R. I. Mohamed, "A novel and generalized three-phase power flow algorithm for islanded microgrids using a Newton trust region method," IEEE Trans. Power Syst., vol. 28, no. 1, pp. 190-201, Feb. 2013.

[8] M. M. A. Abdelaziz and E. F. El-Saadany, "Voltage and reactive power impacts on successful operation of islanded microgrids," IEEE Trans. Power Syst., vol. 28, no. 2, pp. 1716-1727, May 2013.

[9] M. M. A. Abdelaziz, H. E. Farag, and E. F. El-Saadany, "Optimum droop parameter settings of islanded microgrids with renewable energy resources," IEEE Trans. Sustain. Energy, vol. 5, no. 2, pp. 434-445, Apr. 2014.

[10] E. Bompard, E. Carpaneto, G. Chicco, and R. Napoli, "Convergence of the backward/forward sweep method for the load-flow analysis of radial distribution systems," Int. J. Elect. Power Energy Syst., vol. 22, no. 7, pp. 521-530, Oct. 2000.

[11] U. Eminoglu and M. H. Hocaoglu, "Distribution systems forward/backward sweep-based power flow algorithms: A review and comparison study," Elect. Power Compon. Syst., vol. 37, no. 1, pp. 91-110, Dec. 2008.

[12] H. Nikkhajoei and R. Iravani, "Steady-state model and power flow analysis of electronically-coupled distributed resource units," IEEE Trans. Power Del., vol. 22, no. 1, pp. 721-728, Jan. 2007.

[13] M. Z. Kamh and R. Iravani, "Unbalanced model and power-flow analysis of microgrids and active distribution systems," IEEE Trans. Power Del., vol. 25, no. 4, pp. 2851-2858, Oct. 2010.

[14] D. Shirmohammadi, H. Hong, A. Semlyen, and G. Luo, "A compensation-based power flow method for weakly meshed distribution and transmission networks," IEEE Trans. Power Syst., vol. 3, no. 2, pp. 753-762, May 1988.

[15] A. Dukpa, B. Venkatesh, and M. El-Hawary, "Application of continuation power flow method in radial distribution systems," Elect. Power Syst. Res., vol. 79, no. 11, pp. 1503-1510, Nov. 2009.

[16] A. Gómez-Expósito, A. Conejo, and C. Cañizares, Electric Energy Systems: Analysis and Operation. Boca Raton, FL, USA: CRC Press, 2008.

[17] A. Augugliaro, L. Dusonchet, S. Favuzza, M. Ippolito, and E. R. Sanseverino, "A backward sweep method for power flow solution in distribution networks," Int. J. Elect. Power Energy Syst., vol. 32, no. 4, pp. 271-280, May 2010.

[18] G. Luo and A. Semlyen, "Efficient load flow for large weakly meshed networks," IEEE Trans. Power Syst., vol. 5, no. 4, pp. 1309-1316, Nov. 1990.

[19] A. Lisboa, L. Guedes, D. Vieira, and R. Saldanha, "A fast power flow method for radial networks with linear storage and no matrix inversions," Int. J. Elect. Power Energy Syst., vol. 63, pp. 901-907, Dec. 2014.

[20] J.-H. Teng, "A direct approach for distribution system load flow solutions," IEEE Trans. Power Del., vol. 18, no. 3, pp. 882-887, Jul. 2003.

[21] G. Díaz, C. González-Morán, and C. Viescas, "Operating point of islanded microgrids consisting of conventional doubly fed induction generators and distributed supporting units," IET Renew. Power Gener. vol. 6, no. 5, pp. 303-314, Sep. 2012.

[22] G. Diaz, C. Gonzalez-Moran, J. Gomez-Aleixandre, and A. Diez, "Complex-valued state matrices for simple representation of large autonomous microgrids supplied by $\mathrm{PQ}$ and $\mathrm{Vf}$ generation," IEEE Trans. Power Syst., vol. 24, no. 4, pp. 1720-1730, Nov. 2009. 
[23] M. Baran and F. Wu, "Network reconfiguration in distribution systems for loss reduction and load balancing," IEEE Trans. Power Del., vol. 4, no. 2, pp. 1401-1407, Apr. 1989.

[24] R. Srinivasa Rao, S. V. L. Narasimham, M. Ramalinga Raju, and A. Srinivasa Rao, "Optimal network reconfiguration of large-scale distribution system using harmony search algorithm," IEEE Trans. Power Syst., vol. 26, no. 3, pp. 1080-1088, Aug. 2011.

[25] G. Diaz, C. Gonzalez-Moran, J. Gomez-Aleixandre, and A. Diez, "Scheduling of droop coefficients for frequency and voltage regulation in isolated microgrids," IEEE Trans. Power Syst., vol. 25, no. 1, pp. 489-496, Feb. 2010.

Guzmán Díaz (M’04-SM'12) received the M.Eng. and Ph.D. degrees from the University of Oviedo, Gijón, Spain, in 1993 and 2001, respectively, both in electrical engineering.

He is currently an Associated Professor with the Department of Electrical Engineering, University of Oviedo. His current research interests include distributed generation and stochastic analysis.
Javier Gómez-Aleixandre (M'94) received the M.Eng. degree from the Politechnical University of Madrid, Madrid, Spain, in 1984, and the Ph.D. degree from the University of Oviedo, Gijón, Spain, in 1988, both in electrical engineering.

He is currently a Full Professor with the Department of Electrical Engineering, University of Oviedo. He has been the Head of several research projects in his research fields. His current research interests include optimization of asynchronous motors and microgrids, and analysis of faulted electric machines.

José Coto received the M.Sc. degree in electronic and automatic control engineering, and the Ph.D. degree in electric engineering from the University of Oviedo, Gijón, Spain, in 1994 and 2001, respectively.

From 1994 to 1998, he was a Junior Engineer with different electric Spanish utilities. He was a Researcher with the Electric Division, University of Oviedo, where he developed his thesis, and where since 2001, he has been an Associate Professor with the Electric Engineering Department. $\mathrm{He}$ was a Manager of the Scientific-Technical Services and Big Laboratories in Organizational Structure at University of Oviedo, for the last three years. His current research interests include electricity markets, smart grids, distribution, and transmission system operation, where he develops several research projects with the main Spanish power companies. 